\title{
Two-dimensional noise-sustained structures in optical parametric oscillators
}

\author{
Marco Santagiustina, Pere Colet, Maxi San Miguel, and Daniel Walgraef* \\ Instituto Mediterraneo de Estudios Avanzados, IMEDEA ${ }^{\dagger}$ (CSIC-UIB), E-07071 Palma de Mallorca, Spain
}

(Received 17 February 1998)

\begin{abstract}
The problem of two-dimensional (2D), transverse, noise-sustained pattern formation is theoretically and numerically studied, in the case of an optical parametric oscillator, for negative signal detuning. This gives a complete analysis of a $2 \mathrm{D}$, convective, pattern forming system which is also relevant to more general $2 \mathrm{D}$ physical systems. For the optical parametric oscillator, the transversal walk-off due to the nonlinear crystal birefringence, exploited to phase match the frequency down-conversion process, turns the instability to convective up to a certain threshold. In this regime, noise-sustained patterns can be observed. These structures are a macroscopic manifestation of amplified microscopic noise which, in the context of optics, can be of quantum nature. Directly observable properties of the near and far field as well as statistical properties of the spectral intensity help to distinguish noise- from dynamics-sustained structures. Moreover, the analysis indicates that the walk-off term breaks the rotational symmetry of the 2D model. This causes a preferential selection of the stripe orientation, which would be otherwise random, the modulus of the wave vector being the only restricted value. At the convective threshold an entire set of spatial modes becomes unstable, whereas the threshold of absolute instability depends on the relative orientation of the mode. Beyond the threshold for absolute instability, this causes the coexistence, in the linear regime of evolution, of modes that are absolutely unstable, and others that are only convectively unstable. The numerical solutions of the dynamical equations of the system under study confirm the analytical predictions for the value of the instability thresholds and the kind of pattern selected. Moreover, they allow us to investigate the nonlinear regime showing qualitatively the coexistence of modes with different types of instability and giving a quantitative characterization of the transition from noise-sustained to dynamics-sustained structures. [S1063-651X(98)14308-8]

PACS number(s): 42.65.Sf, 42.50.-p, 42.65.Yj
\end{abstract}

\section{INTRODUCTION}

Pattern formation in nonlinear optical systems is the object of an intense investigation, both theoretical and experimental [1]. Spontaneous pattern formation has been observed or predicted in many different nonlinear optical systems such as nonlinear Kerr media in resonators [2,3], Kerr slices in single mirror feedback configuration [4-7], two-level atoms [8], atomic and molecular gas media $[9,10]$, second harmonic generation [11], and optical parametric oscillators [12,13]. The range of observable patterns and parameter range of observation are highly enhanced when considering the extra degree of freedom associated with the polarization of light [14-17]. Stripes, squares, hexagons, and more complicated stationary and dynamical patterns have been observed and characterized in the publications. A description of many of these phenomena in terms of universal amplitude equations gives the link which interconnects the optical patterns with those similarly observed in other systems [18].

Nowadays, optical parametric oscillators (OPO's) are among the most attracting optical devices where pattern formation is studied $[12,13,19,20]$, and the recent experimental observation [21] of spatial patterns in quadratic media is very

\footnotetext{
*Permanent address: Center for Nonlinear Phenomena and Complex Systems, Université Libre de Bruxelles, Campus Plaine, Boulevard du Triomphe B.P. 231, 1050 Bruxelles, Belgium.

${ }^{\dagger}$ URL: http://www.imedea.uib.es/PhysDept
}

encouraging. This interest stems from the foreseen applications in the field of all-optical processing and storage of information [1], as well as from the fact that OPO's can generate squeezed [22] and other nonclassical states of light [23]. For the latter reason they are thought to be a paradigmatic example of the interface between classical and macroscopic quantum patterns [24]. In this regard, there are recent studies which focus mostly on the quantum correlations associated with a pattern forming instability [24-29]. There, particular attention is paid to the noisy precursors observed below the threshold of the instability, because they are spatially organized manifestations of quantum fluctuations. This situation is, from the classical viewpoint, conceptually equivalent to the convection experiments of Ref. [30], aimed at characterizing thermal fluctuations. In both cases spatial correlation are investigated very close to the instability threshold where fluctuations are weakly damped. In the classical case, features in the correlation functions such as noise squeezing under the minimum classical level are obviously absent. The analogy with other experiments in classical fluid dynamics [31] has suggested [32] that nonlinear optical systems can present macroscopic, noise-sustained structures, above the threshold of the instability in the so-called convectively unstable regime [33]. This phenomenon refers to the situation when local perturbations of the steady state can be advected much rapidly than their rate of spreading. In this case macroscopic patterns can arise and be observed only if noise is continuously applied, the pattern now being regenerated at any time, hence the name noise-sustained structures. These structures are the result of noise amplification, with magnification factors of several order of magnitudes. 
They are thus interesting candidates for the study of quantum correlations in spatially structured systems. This situation is distinct from that of the noisy precursors, where noise is selectively enhanced, but not amplified, by the nonlinear spatial filtering effect.

Noise-sustained structures were predicted and characterized in optical passive cavities filled with a nonlinear Kerr medium and pumped by a tilted external beam [32]. In this situation the advection motion is induced by input pump beam tilting [34]. Here we show that the convectively unstable regime and thus noise-sustained structures are also found in type-I degenerate OPO's for positive signal detunings. The present extension includes important features which are either peculiar to the OPO or of wider interest in the study of convective instabilities in other physical systems.

In this paper a transversal two-dimensional (2D) model is considered for the OPO. Note that the 1D model of Ref. [32] was justified by both theoretical [2,34] and experimental [6] results on the drift instability in nonlinear Kerr devices similar to the one we considered. For the OPO this simplification is generally avoided, and the investigation is directed toward 2D transverse devices $[12,13,19,20]$. We want to stress that, in spite of the particular choice of the OPO, the features that are found belong very generally to the whole class of 2D, convective, pattern forming systems. To the best of our knowledge the generation of noise-sustained structures was never studied before in a 2D system, though the theory of convective and absolute instability in higher-dimensional systems was developed in plasma physics $[35,36]$. We will show that pattern formation is highly affected by the advection term in the convectively unstable regime and also that this is an important result in the absolutely unstable regime. In fact, the advection breaks the rotational symmetry of the system, and this causes a preferential orientation of the stripes, which can be explained only if the analysis is carried out taking into account the possible convective nature of the instability.

It is also worth noting that in the specific case of OPO's the convective regime can hardly be neglected because birefringent crystals are used to phase match the downconversion process. As known, the anisotropy of the medium implies that a transverse walk-off effect among the beams, due to the misalignment of the Poynting vectors [37-39], can occur. This means that an advection term is present in the governing equation, even when the pump beam is aligned with the cavity. Moreover the typical walk-off angle can often be larger than a few mrad and thus this effect is expected to overcome finite-size locking effects too [40].

This paper is organized as follows. In Sec. II we present the model used to represent a type-I degenerate OPO. Section III is dedicated to a linear stability analysis, which takes into account the possible convective nature of the instability. In Sec. IV, we analyze the generation of the noise-sustained convective structures and characterize the typical features of the pattern. A discussion about the possible the experimental observation of such structures and the conclusions are presented in Sec. V.

\section{GOVERNING EQUATIONS}

In this section we introduce the set of semiclassical equations we use to model the OPO. Such a device may consist,

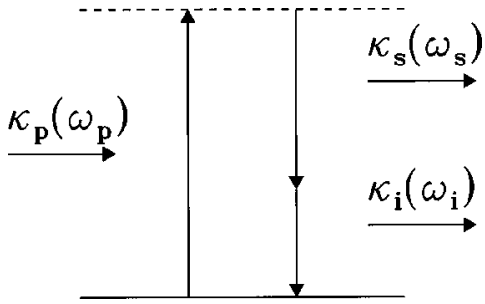

FIG. 1. Schematic representation of the frequency downconversion process

for example, of a ring resonator with flat mirrors, filled with a quadratic $\left(\chi^{(2)}\right)$ nonlinear medium and pumped by an external laser source of frequency $\omega_{p}$. The output mirror allows a fraction of the internal field to leak out, for detection, in the near field (NF, i.e., close to the output mirror) and far field (FF, far from the device) configurations. In this system the FF is the spatial Fourier transform of the NF. Under suitable experimental conditions (which we will soon specify) together with the residual input beam at $\omega_{p}$, two additional frequency components $\omega_{s}$ and $\omega_{i}$ (usually called the signal and the idler) can be detected at the output port. They are generated by the nonlinear interaction which takes place inside the crystal: light quanta of the pump beam are down-converted to $\omega_{s}$ (signal) and $\omega_{i}$ (idler) photons via the parametric process, which is sketched in Fig. 1. This process is highly efficient when two conditions are satisfied [37,39]:

$$
\begin{gathered}
\omega_{p}=\omega_{s}+\omega_{i} \\
\kappa_{p}\left(\omega_{p}\right)=\kappa_{s}\left(\omega_{s}\right)+\kappa_{i}\left(\omega_{i}\right) .
\end{gathered}
$$

These represent, respectively, the conservation of the energy and momentum [41] of the photons involved in the interaction. In the remainder of the paper we will assume the downconversion process to be frequency degenerated, i.e. $\omega_{s}$ $=\omega_{i}=\omega_{0}$ (hereafter the fundamental harmonic, $\mathrm{FH}$ ). Hence Eq. (1) implies that $\omega_{p}=2 \omega_{0}$ (hereafter the second harmonic, $\mathrm{SH}$, or simply the pump).

As for Eq. (2), it means that the process requires a phasematching condition for the wave vectors which can be rewritten (taking into account the frequency degeneracy) in the form

$$
n_{p}\left(2 \omega_{0}\right)=\frac{n_{s}\left(\omega_{0}\right)+n_{i}\left(\omega_{0}\right)}{2}
$$

where $n_{p, s, i}(\omega)$ are the refractive indexes of the pump, the signal, and the idler, which in general depend on both the frequency and the polarization of the fields. The phasematching condition (3) cannot be trivially satisfied for an arbitrary choice of the frequency and of the nonlinear medium. Quite often birefringence is exploited, since it allows one to compensate for the index frequency dispersion by means of orthogonally polarized waves $[37,39]$. In fact, in a uniaxial birefringent crystal there exist two preferential orthogonal linear polarizations, propagating independently with a different refractive index (for a discussion of the normal modes of a birefringent medium; see, for example Ref. [42]). Thus there are two types of phase-matching conditions $[37,39]$ : one involves polarization nondegenerate, down- 
converted beams, i.e., the polarization of $\omega_{s}$ is orthogonal to that of $\omega_{i}$ and is usually referred to as the type-II matching. The type-I phase matching, conversely, involves polarization degenerate output photons, i.e., the signal and the idler have the same polarization which is orthogonal to that of the pump in order to satisfy Eq. (3). We will consider only this second case, because type-I phase matching is a common experimental setup in second harmonic generation (SHG) and OPO's $[37,39,43]$, and because this reduces the number of equations which govern the phenomenon. In this case

$$
n_{p}\left(2 \omega_{0}\right)=n_{s}\left(\omega_{0}\right)=n_{i}\left(\omega_{0}\right)=n .
$$

The aim of reviewing these well known features of the parametric down-conversion is to stress that this process is commonly obtained by means of birefringent media and orthogonally polarized beams, a fact that brings important consequences. In fact, except for the particular case in which the propagation takes place along an optical axis (noncritical phase matching), one wave is no longer polarized in a normal mode. It can be demonstrated that for this beam, called extraordinary, the Poynting vector is not parallel to the propagation direction (again see Ref. [42] for details). Conversely, the other orthogonally polarized beam still has its Poynting vector parallel to the propagation direction, and is thus defined as an ordinary ray. Therefore, the ordinary and extraordinary beams go slightly misaligned during propagation, and they walk off one from each other [37-39,42].

The walk-off effect, though considered for modeling pulse generation in OPO's [44] and solitary wave propagation in SHG [45], has been neglected in the previous analyses of transverse structure formations in the OPO's. In principle it seems logical that this term could be neglected for its smallness in the noncritical or quasi-non-critical phase matching (propagation direction close to an optical axis). However, in the more general case and despite its smallness, this term can be of fundamental importance as soon as the presence of noise is considered. The effect of the transverse walk-off is in fact equivalent, as it appears in the dynamical equations, to the pump beam tilting of the Kerr case [34]. As demonstrated in Ref. [32], a convection term, like the one introduced by beam tilting or walk-off effects, gives rise to a convectively unstable regime, where noise-sustained structures can be observed. Moreover, we will show that in a 2D system this term causes a preferential pattern orientation in the absolutely unstable regime. This effect can be explained only by taking into account the mode selection mechanism introduced by the convectionlike term.

The equations governing the time evolution of the $\mathrm{SH}$ and FH field envelopes in the resonator can be obtained in two steps. First we can derive, in the slowly varying envelope approximation (SVEA), the propagation equations in the nonlinear medium, for example by means of a multiple scales expansion (see also Ref. [39]). Then, by following the guidelines of Ref. [8] we can find, in the mean-field limit (MFL), the time evolution equations when the medium fills a ring resonator. In a ring cavity, photons are generated in the crystal and moves transversally with a walk-off angle that can be typically of the order of $0.1 \mathrm{mrad}$. So the actual displacement attained after the propagation in the crystal is very small. Outside the crystal the waves are parallel, and thus the signal generated is reinjected collinearly with the pump at the next pass, but slightly displaced in the walk-off direction. The mean-field approximation is thus averaging out the effect of this continuous space displacement of photons. We have performed all steps and finally obtained the following equations for the envelopes of the SH $\left(A_{0}(x, y, t)\right.$, ordinary polarized) and $\mathrm{FH}\left(A_{1}(x, y, t)\right.$, extraordinary polarized) (see also Refs. $[12,13,19,46])$ :

$$
\begin{aligned}
& \partial_{t} A_{0}= \gamma_{0}\left[-\left(1+i \Delta_{0}\right) A_{0}+E_{0}+i a_{0} \nabla^{2} A_{0}+2 i K_{0} A_{1}^{2}\right] \\
&+\sqrt{\epsilon_{0}} \xi_{0}(x, y, t) \\
& \partial_{t} A_{1}= \gamma_{1}\left[-\left(1+i \Delta_{1}\right) A_{1}+\rho_{1} \partial_{y} A_{1}+i a_{1} \nabla^{2} A_{1}+i K_{0} A_{1}^{*} A_{0}\right] \\
&+\sqrt{\epsilon_{1}} \xi_{1}(x, y, t)
\end{aligned}
$$

where $x$ and $y$ are the transversal spatial dimensions, $t$ the time, and the coefficients are defined as follows. The decay rates of the $\mathrm{SH}$ and $\mathrm{FH}$ fields in the cavity are $\gamma_{0,1}$ $=v_{0,1} T_{0,1} / L$, where $v_{0,1}$ are their group velocities, $T_{0}=T_{1}$ $=T \ll 1$ are the output mirror transmission coefficients, and $L$ is the cavity length. The scaled cavity detunings are

$$
\begin{gathered}
\Delta_{0}=\left(\omega_{c 0}-2 \omega_{0}\right) / \gamma_{0}-\Delta \kappa L / T_{0}, \\
\Delta_{1}=\left(\omega_{c 1}-\omega_{0}\right) / \gamma_{1},
\end{gathered}
$$

where $\omega_{c 0, c 1}$ are the cavity resonances closest to the SH and FH frequencies. The second term in Eq. (7) takes into account a possible phase mismatch $\Delta \kappa$ of the parametric interaction. In order to be consistent with the MFL this term must be small, i.e., $\Delta \kappa \ll 1 / L$, so it can be included in the detuning parameter as shown. Note also that the bandwidth of the phase matching is much larger that the cavity modes and thus, if present, the mismatch can be considered constant. Therefore, the limits of validity of this model are those set by the SVEA-MFL approximation (see Ref. [8] for a detailed definition) plus the additional condition of a small phase mismatch. Here, for the sake of simplicity, we will suppose that Eq. (4) holds and thus $\Delta \kappa=0$. The coefficients $a_{0,1}$ $=L /\left(2 \kappa_{0,1} T_{0,1}\right)$, where $\kappa_{0,1}=\kappa_{p, s}$, represent diffraction. They are related by $a_{0}=a_{1} / 2$ since $\kappa_{0}=2 \kappa_{1}$ as a consequence of Eq. (4) and the frequency degeneracy, and because we have previously set $T_{0}=T_{1}=T$. The coefficient $\rho_{1}$ $=L \tan \left(\alpha_{1}\right) / T_{1}$, where $\alpha_{1}$ is the angle between the SH and FH Poynting vectors induced by the birefringence $[37,39,42,45]$, is the walk-off parameter. The term $E_{0}(x, y, t)$ is the input SH pump and $K_{0}=\omega_{0} \chi_{\mathrm{eff}}^{(2)} L /(n c T)$ the nonlinear coefficient, where $\chi_{\text {eff }}^{(2)}$ is the effective (quadratic) susceptibility and $n$ is defined by Eq. (4).

Finally, the last terms in the equations are complex, Gaussian white noise with zero mean $\left(\left\langle\xi_{0,1}(x, y, t)\right\rangle=0\right)$ and correlations $\left\langle\xi_{i}(x, y, t) \quad \xi_{j}^{*}\left(x^{\prime}, y^{\prime}, t^{\prime}\right)\right\rangle=2 \delta_{i, j} \delta\left(t-t^{\prime}\right) \delta(x$ $\left.-x^{\prime}\right) \delta\left(y-y^{\prime}\right) \quad i, j=0,1$. In a linearized version of Eqs. (5), and (6), they describe quantum noise in the Wigner representation, as shown in Ref. [28]. Here they can account for thermal and input field fluctuations as well. 

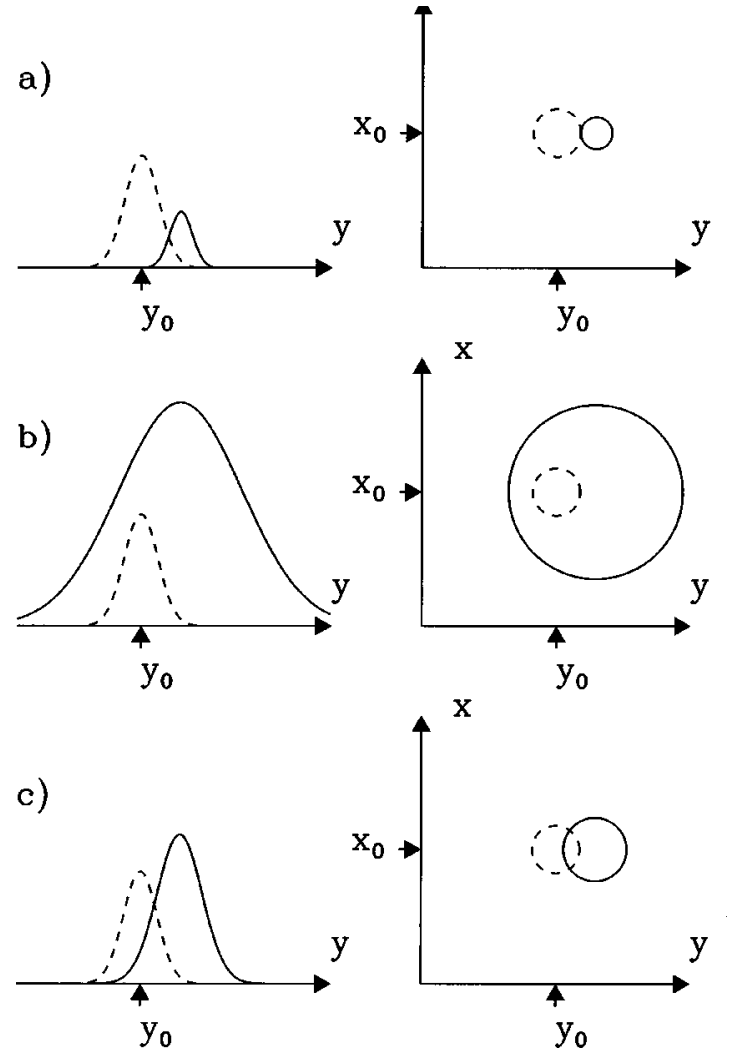

FIG. 2. Pictorial definition of the stable and unstable regimes in a two-dimensional system with a transversal walk-off. The left (right) column is a lateral (top) view of a perturbation of the steady state. Dashed (solid) curves represent the $t=0(t>0)$ conditions for (a) the stable, (b) the absolutely unstable, and (c) the convectively unstable regimes. At the fixed position $\vec{r}_{0}=\left(x_{0}, y_{0}\right)$ the field amplitude (a) decays, (b) grows, and (c) decays due to the strong advection compared to spread.

\section{CONVECTIVE AND ABSOLUTE LINEAR INSTABILITY ANALYSIS}

This section presents the linear instability analysis of the steady-state solution of Eqs. (5) and (6) which corresponds to the OPO below the threshold of signal generation. This will be carried out according to the theory presented in Refs. $[33,35,47]$ in order to be able to discriminate convective from absolute instabilities.

For the sake of clarity, we first recall the definition of the convective instability for a $2 \mathrm{D}$ system. In general, a steady state is defined to be absolutely stable (unstable) if a localized perturbation decays (grows) with time. In Fig. 2(a) and 2(b), we show these two situations for a system in which advection is also present along the $y$ axis. However, there is a third possibility: the perturbation may grow (unstable) but the advection velocity could overwhelm the speed of spreading in the direction of the advection [Fig. 2(c)]. In this case the perturbation eventually leaves the system which returns to the steady state. This type of instability is defined to be convective.

Clearly, the definition depends on the choice of the system of reference; in fact, by choosing a reference frame moving at the speed of the perturbation peak we would always define a system to be absolutely stable or unstable. However, in finite physical systems, there is always a preferred refer- ence frame, which makes the above definition unambiguous. In the OPO, for example, the pump laser beam is present in a well defined region of space, and thus it can be taken as the fixed frame. The FH field moves transversally due to the walk-off effect, and thus a perturbation of the FH might leave the pump region at some time. Clearly, outside that portion of space there is no amplification via the downconversion process, and the $\mathrm{FH}$ fades because of mirror losses.

Thus we proceed by analyzing the parameter range for which the system of equations (5) and (6) is stable, convectively unstable, or absolutely unstable. When $\epsilon_{0,1}=0$, the equations have the following uniform steady state:

$$
A_{0}=\frac{E_{0}}{1+i \Delta_{0}}, \quad A_{1}=0
$$

To determine when the latter becomes unstable we can linearize Eqs. (5) and (6) close to the steady state, and look for solutions of the kind $\exp (i \vec{q} \cdot \vec{r}+\lambda t)$, where $\vec{q}$ is a twodimensional vector with real components, $\vec{q}=\left(q_{x}, q_{y}\right) \in \mathcal{R}^{2}$ and $\vec{r}=(x, y)$. It turns out that the steady state becomes unstable only along the FH component $\left(A_{1}\right)$ of the eigenvector and thus the analysis reduces to the study of the linearized form of Eq. (6) with $A_{0}$ given by Eq. (9) $[12,13,19,46]$. The dispersion relation obtained is

$$
\lambda_{ \pm}(\vec{q})=i q_{y} \rho_{1}-1 \pm \sqrt{F^{2}-\left[\Delta_{1}+a_{1}\left(q_{x}^{2}+q_{y}^{2}\right)\right]^{2}},
$$

where $F^{2}=K_{0}^{2}\left|E_{0}\right|^{2} /\left(1+\left|\Delta_{0}\right|^{2}\right)$ is a normalized pump intensity.

The determination of the nature of the instability entails the estimation of the linearized asymptotic behavior of a generic perturbation of the FH steady state, say $\psi$, which is given by

$$
\begin{aligned}
\psi(x, y, t)= & \int_{-\infty}^{+\infty} \int_{-\infty}^{+\infty} d q_{x} d q_{y} \widetilde{\psi}\left(q_{x}, q_{y}, 0\right) \exp \left[i\left(q_{x} x+q_{y} y\right)\right. \\
& \left.+\lambda\left(q_{x}, q_{y}\right) t\right]
\end{aligned}
$$

where $\widetilde{\psi}\left(q_{x}, q_{y}, 0\right)$ is the initial perturbation in the spatial wave-vector space, and $\lambda\left(q_{x}, q_{y}\right)$ is the eigenvalue with largest real part (plus sign).

According to the definition given above (Fig. 2), the system is (absolutely) stable when $\left|\psi\left(\vec{r}_{0}+\vec{v} t, t\right)\right| \rightarrow 0$ as $t \rightarrow \infty$ for any arbitrary fixed $\vec{r}_{0}$ and any velocity $\vec{v}$ [33]. It is easy to demonstrate that the condition to have absolute stability is $\operatorname{Re}\left(\lambda\left(q_{x}, q_{y}\right)\right)<0$ for any $\left(q_{x}, q_{y}\right) \in \mathcal{R}^{2}$, because it yields an integral (11) decaying with time.

The modes $\left(q_{x}^{m}, q_{y}^{m}\right)$ with the largest growth rate $\operatorname{Re}(\lambda)$ are those of modulus

$$
\left|\left(q_{x}^{m}, q_{y}^{m}\right)\right|=q_{c}=\sqrt{-\frac{\Delta_{1}}{a_{1}}}
$$

if $\Delta_{1}<0$, and $\left(q_{x}^{m}, q_{y}^{m}\right)=(0,0)$ otherwise. In the following we treat the case $\Delta_{1}<0$ where the maximum of $\operatorname{Re}(\lambda)$ is reached on a ring of modes in the wave-vector space [48]. The threshold for parametric down-conversion takes place 
when $\operatorname{Re}(\lambda)=0$, i.e., when $F^{2}=1$, and above threshold formation of a stripe pattern is expected $[12,13,19,20]$.

When $\operatorname{Re}\left(\lambda\left(q_{x}, q_{y}\right)\right)>0$ for some wave vector $\left(q_{x}, q_{y}\right)$, there can be two situations. We can either have that $|\psi(\vec{r}, t)| \rightarrow \infty$ for any arbitrary value of $\vec{r}$ (absolutely unstable) or that $\left|\psi\left(\vec{r}_{0}, t\right)\right| \rightarrow 0$ and $\left|\psi\left(\vec{r}_{0}+\vec{v} t, t\right)\right| \rightarrow \infty$ for some velocity $\vec{v}$ and any arbitrary $\vec{r}_{0}$ (convectively unstable). Note that for determining the asymptotic behavior of the perturbation we only need to evaluate integral (11) for those values of $\left(q_{x}, q_{y}\right)$ for which $\operatorname{Re}\left(\lambda\left(q_{x}, q_{y}\right)\right) \geqslant 0$, the other modes giving no contribution (they are surely decaying). The extrema of the surface of integration are thus identified by the loci in the real plane $\left(q_{x}, q_{y}\right)$ where $\operatorname{Re}(\lambda)=0$. In order to illustrate the integration technique, we first consider the analysis with only one spatial dimension $\vec{q} \rightarrow q$. The complex function of real variable $\lambda(q)$ can be analytically continued over the complex plane $k$ in the form $\lambda(k / i)$. The previous real domain of definition corresponds to a purely imaginary $k$. Then, using the Cauchy theorem, we can equivalently evaluate Eq. (11) along any contour in the complex wave-vector plane connecting the extrema of integration, provided that the integrand has no singularities in the area bounded by the original and the new contour. In particular we can evaluate it for a contour which crosses a saddle point $k_{s}$ in the complex plane, along the direction of the steepest descent. The asymptotic behavior of the integral is then given by the value of the exponential part of the integrand calculated at the saddle point. Eventually, if $\operatorname{Re}\left(\lambda\left(k_{s}\right)\right)>0$ this term diverges and the instability is absolute (see an example in one dimension, Taylor-Couette flows in Ref. [49]). In two dimensions we can proceed in a similar way and the integral yields an absolutely unstable solution if the following conditions are satisfied:

$$
\begin{gathered}
\operatorname{Re}\left(\lambda\left(k_{x}^{s}, k_{y}^{s}\right)\right)>0, \\
\operatorname{Re}\left(\left.\nabla_{\vec{k}}^{2} \lambda\left(k_{x}, k_{y}\right)\right|_{\left.k_{x}^{s}, k_{y}^{s}\right) \geqslant 0,}\right.
\end{gathered}
$$

where $\left(k_{x}^{s}, k_{y}^{s}\right)$, defined by

$$
\left.\nabla_{\vec{k}} \lambda\left(k_{x}, k_{y}\right)\right|_{k_{x}^{s}, k_{y}^{s}}+i \frac{\vec{r}}{t}=0,
$$

is the saddle point in the $\vec{k}$ complex space [35]. Taking $\vec{r}$ $=\vec{r}_{0}+\vec{v} t$, for $t \rightarrow \infty$ the saddle is given by $\left.\nabla_{\vec{k}} \lambda\left(k_{x}, k_{y}\right)\right|_{k_{x}^{s}, k_{y}^{s}}$ $+i \vec{v}=0$. In the fixed reference frame of the pump beam, $\vec{v}$ $=0$, and so

$$
\left.\nabla_{\vec{k}} \lambda\left(k_{x}, k_{y}\right)\right|_{k_{x}^{s}, k_{y}^{s}}=0 \text {. }
$$

To summarize: if $\operatorname{Re}\left(\lambda\left(q_{x}^{m}, q_{y}^{m}\right)\right)<0$, where $\left(q_{x}^{m}, q_{y}^{m}\right)$ is the loci (in $\mathcal{R}^{2}$ ) of the maxima of $\operatorname{Re}(\lambda)$ the system is absolutely stable; if $\operatorname{Re}\left(\lambda\left(q_{x}^{m}, q_{y}^{m}\right)\right)>0$ and $\operatorname{Re}\left(\lambda\left(k_{x}^{s}, k_{y}^{s}\right)\right)<0$ where $\left(k_{x}^{s}, k_{y}^{s}\right)$ is the saddle point (in $\left.\mathcal{C}^{2}\right)$, it is convectively unstable and it is absolutely unstable if $\operatorname{Re}\left(\lambda\left(k_{x}^{s}, k_{y}^{s}\right)\right)>0$.

For the OPO, by replacing $\left(q_{x}, q_{y}\right)$ with $\left(k_{x}, k_{y}\right) / i$, and taking into account Eq. (12), we can rewrite $\lambda_{+}$of Eq. (10) as

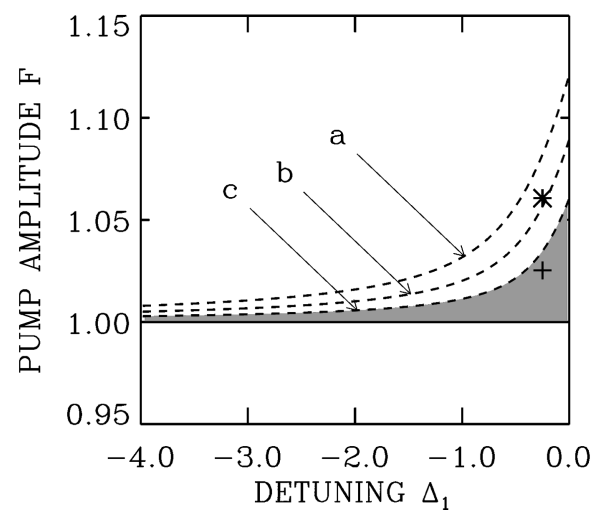

FIG. 3. Stability diagram as a function of the FH detuning for the steady-state solution (9). In the region below the solid line the solution is always stable; the dashed curves represent the absolute instability thresholds for different values of the walk-off parameter: (a) $\rho_{1}=0.25$, (b) $\rho_{1}=0.2$, and (c) $\rho_{1}=0.15$. The region between the solid and dashed lines is the domain of convective instability for a given value of $\rho_{1}$. In particular, we shadowed the region referring to $\rho_{1}=0.15$ (other parameters: $\gamma_{0}=\gamma_{1}=1$ and $a_{1}=0.25$ ). The star $(*)$ and the plus $(+)$ signs indicate the parameters used for the numerical solutions of Figs. 4 and 5, respectively.

$$
\lambda\left(k_{x}, k_{y}\right)=-1+\rho_{1} k_{y}+\sqrt{F^{2}-a_{1}^{2}\left(q_{c}^{2}+k_{x}^{2}+k_{y}^{2}\right)^{2}},
$$

and thus the saddle is found through Eq. (16) at

$$
\begin{gathered}
\frac{2 a_{1}^{2}\left[q_{c}^{2}+\left(k_{x}^{s}\right)^{2}+\left(k_{y}^{s}\right)^{2}\right] k_{x}^{s}}{\sqrt{F^{2}-a_{1}^{2}\left[q_{c}^{2}+\left(k_{x}^{s}\right)^{2}+\left(k_{y}^{s}\right]^{2}\right)^{2}}}=0, \\
\rho_{1}-\frac{2 a_{1}^{2}\left[q_{c}^{2}+\left(k_{x}^{s}\right)^{2}+\left(k_{y}^{s}\right)^{2}\right] k_{y}^{s}}{\sqrt{F^{2}-a_{1}^{2}\left[q_{c}^{2}+\left(k_{x}^{s}\right)^{2}+\left(k_{y}^{s}\right]^{2}\right)^{2}}}=0,
\end{gathered}
$$

For $\rho_{1}=0$ the saddle solution of Eq. (18) coincides with Eq. (12), and therefore the criterion of convective and absolute instability coincides. For $\rho_{1} \neq 0$, the saddle is given by

$$
k_{x}^{s}=0, \quad \rho_{1}-\frac{2 a_{1}^{2}\left[q_{c}^{2}+\left(k_{y}^{s}\right)^{2}\right] k_{y}^{s}}{\sqrt{F^{2}-a_{1}^{2}\left[q_{c}^{2}+\left(k_{y}^{s}\right)^{2}\right]^{2}}}=0 .
$$

The second of Eq. (19) was numerically resolved for a complex $k_{y}^{s}$ together with $\operatorname{Re}\left(\lambda\left(0, k_{y}^{s}\right)\right)=0$ to calculate the value of $F$ at the absolute instability threshold. The result is shown, as a function of the FH detuning $\Delta_{1}$, in Fig. 3 .

When the OPO is at threshold $(F=1)$ and $\rho_{1} \neq 0$, all unstable modes are convective; if the pump amplitude is increased, the first mode to become absolutely unstable satisfies $k_{x}=0$ [the first of Eqs. (19)]. The advection term breaks the rotational symmetry and stripes parallel to the $x$ axis are always the selected mode. In fact, from the value of the saddle given by Eq. (19) the selected wave vector can be determined [50,51]: in particular, we obtain the condition $q_{x}=0$. Note that this asymmetry cannot be ascribed to a change in the growth rate of the most unstable modes which is equal for all the modes on ring (12). The mechanism which creates this selective action is related to the advectionspreading balance peculiar to the convective regime, as we mentioned in Sec. II. Moreover, as at the absolute threshold the only absolutely unstable modes are those close to 
$q_{x}=0$, it is reasonable to assume that the remaining modes should be still convectively unstable. It is then interesting to address the question of the coexistence of absolutely unstable and convectively unstable modes. In the next paragraphs we investigate, through a simple approximated analysis, this phenomenon. The predictions are qualitatively confirmed by numerical solutions which are presented in Sec. IV (see Fig. 6).

First, note that the position of the saddle $\left(k_{x}^{s}, k_{y}^{s}\right)$, and thus of the threshold of the absolute instability, depends on the coefficient of the advection term $\rho_{1}$ [second of Eqs. (19) and Fig. 3]. In particular, the fact that the faster the advection the larger the absolute instability threshold agrees with the intuitive definition we gave of the convective regime (see Fig. 2). The fact that the instability turns to be absolute when the rate of spreading of a perturbation is larger than the advection can be shown by means of a simplified mathematical analysis. Let's consider Eq. (11) again, and make a Taylor expansion of the eigenvalue (10) around a particular wave vector of the ring given by Eq. (12): $q_{x}^{m}=q_{c} \cos \theta, q_{y}^{m}=q_{c} \sin \theta$, where the variable $0<\theta \leqslant \pi / 2$ represents the angle between $\left(q_{x}^{m}, q_{y}^{m}\right)$ and the axis $\left(q_{x}, 0\right)$. If the expansion is truncated at the second order in the differences $q_{x}-q_{x}^{m}$ and $q_{y}-q_{y}^{m}$, the integral (11) can be solved analytically (see the 1D examples in Ref. [47]) and the condition for the absolute instability becomes

$$
\operatorname{Re}\left(\lambda\left(q_{x}^{m}, q_{y}^{m}\right)\right)+\frac{\left(y_{0}+\rho_{1} t\right)^{2}}{2 \operatorname{Re}\left(\lambda_{y y}\left(q_{x}^{m}, q_{y}^{m}\right)\right) t^{2}}>0,
$$

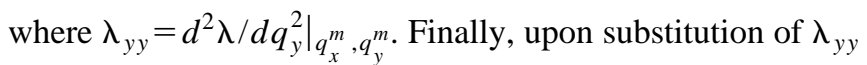
calculated through Eq. (10) and as $t \rightarrow \infty$, we obtain

$$
-1+F\left(1-\frac{\rho_{1}^{2}}{8 a_{1}^{2} q_{c}^{2} \sin ^{2} \theta}\right)>0 .
$$

Equation (21) shows clearly that the larger the group velocity $\rho_{1}$ the larger the threshold value of $F$ for the absolute instability. This approximated solution, valid when the Taylor expansion can be truncated at the second order and for $\theta$ not too close to 0 , is in agreement within a few percent, with the exact resolution given in Fig. 3 for $\theta=\pi / 2$. Moreover, it confirms that the mode $\theta=\pi / 2\left(0, q_{y}\right)$ has the lowest threshold for absolute instability and indicates that for a normalized pump amplitude $F$ above the threshold of absolute instability the modes for which

$$
\theta>\theta_{c}=\arcsin \left(\frac{\rho_{1}}{2 a_{1} q_{c} \sqrt{2}} \sqrt{\frac{F}{F-1}}\right)
$$

are absolutely unstable, while the others are still convectively unstable.

The quantity $\operatorname{Re}\left(\lambda_{y y}\left(q_{x}^{m}, q_{y}^{m}\right)\right) t$ can be interpreted [47] as the mean-square spatial spread of the perturbation in the advection direction. It is then clear that the mode with $\theta$ $=\pi / 2$ spreads faster than the modes with smaller $\theta$, and hence, although all modes on the ring have the same growth rate $\operatorname{Re}\left(\lambda\left(q_{x}^{m}, q_{y}^{m}\right)\right)$, there are some modes which are preferred through a spreading selection mechanism. Eventually, the rotational symmetry is broken.
To summarize, the results of this section are the following. In the OPO's the presence of the walk-off term induces the existence of a convectively unstable regime, just above the OPO threshold of signal generation. In this regime perturbations are advected faster than they spread. The walk-off term does not change the growth rate of modes but breaks the rotational symmetry, because the critical modes $\left(q_{x}^{m}, q_{y}^{m}\right)$ have different spreading velocities in the direction of the advection. This causes the modes with $q_{x}=0$ to be preferentially selected both in the convectively and absolutely unstable regimes. Moreover, the symmetry breaking implies the coexistence of absolutely and convectively unstable modes in a certain region of parameters in the absolutely unstable regime.

Finally, we want to stress that these results, obtained in the specific case of an OPO, are actually very general and could be extended to pattern formation in similar $2 \mathrm{D}$, convective system. To our knowledge, this is the first example of a complete study of the formation of convective structures in $2 \mathrm{D}$ systems.

\section{CHARACTERIZATION OF THE NOISE-SUSTAINED STRUCTURES}

Noise-sustained convective structures, as well as features associated to the $2 \mathrm{D}$ convective model, are presented in this section as they result from the numerical solutions of the dynamical equations (5) and (6). Noise-sustained patterns can be expected in the regime of convective instability, where no dynamics-sustained structure can be observed because of the overwhelming advection. In practice, the noise acts as a perturbation which, being continuously applied, regenerates a new pattern at any time.

We show the integration of Eqs. (5) and (6) with a noise intensity $\epsilon_{0,1}=1.5 \times 10^{-11}$ and with the same integration scheme as in Ref. [32]. The system size was $320 \times 320$ scaled spatial units, and the pump was a super-Gaussian beam: $E_{0}(x, y, t)=E_{m} \exp \left[-\left(\left(x^{2}+y^{2}\right) / \sigma_{0}^{2}\right)^{m} / 2\right]$, with $m=5$ and $\sigma_{0}$ $=112$; the grid was of $512 \times 512$ points and the time step 0.01 normalized units. The super-Gaussian beam is flat top, and this allows us to apply the results of the linear stability analysis which are strictly valid only for a uniform steady state. We also set $\gamma_{0}=\gamma_{1}=1, \Delta_{0}=1, \Delta_{1}=-0.25, a_{0}=a_{1} / 2$ $=0.125, K_{0}=1$, and $\rho_{1}=0.15$. This is equivalent to have scaled, the time with the decay rate, the space with a multiple of the diffraction length, and the amplitude with the nonlinear coefficient. Under this, or similar scaling, the equations become dimensionless; thus all quantities in the figures are dimensionless as well. Note that the parameters of the simulations were chosen in order to make the visualization of the results simpler, but they are not critical. Noise-sustained structures were also found with smaller beams, larger and smaller advection terms, and different SH and FH detunings and diffraction coefficients, close to the one presented.

In the figures we do not show the whole integrating window, but only the central parts. For the NF the spatial region where the generated $\mathrm{FH}$ is appreciably intense $(|x|<103.75,-97.5<y<110)$ is shown; in the FF only the region of low spatial wave vectors around the unstable ring is interesting $\left(\left|q_{x}\right|<1.7,\left|q_{y}\right|<1.7\right)$. Moreover, we did not show the $\mathrm{SH}$, because this field is stable. 
a)

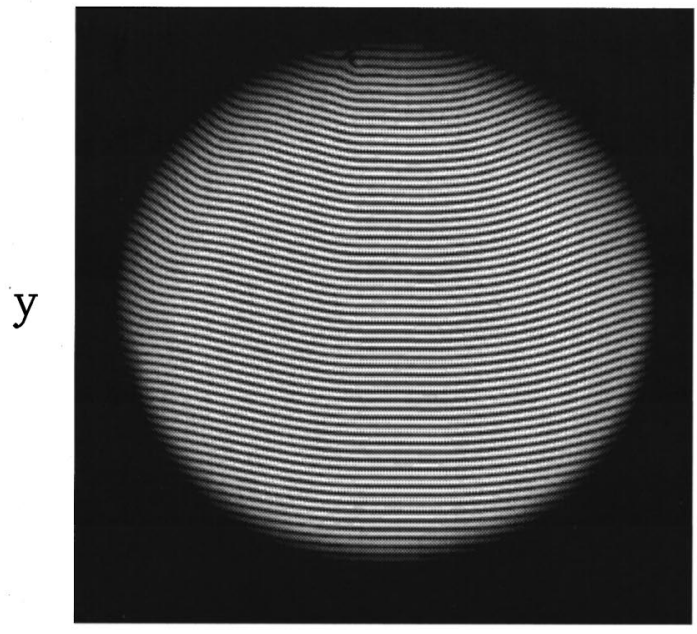

$\mathrm{X}$

b)

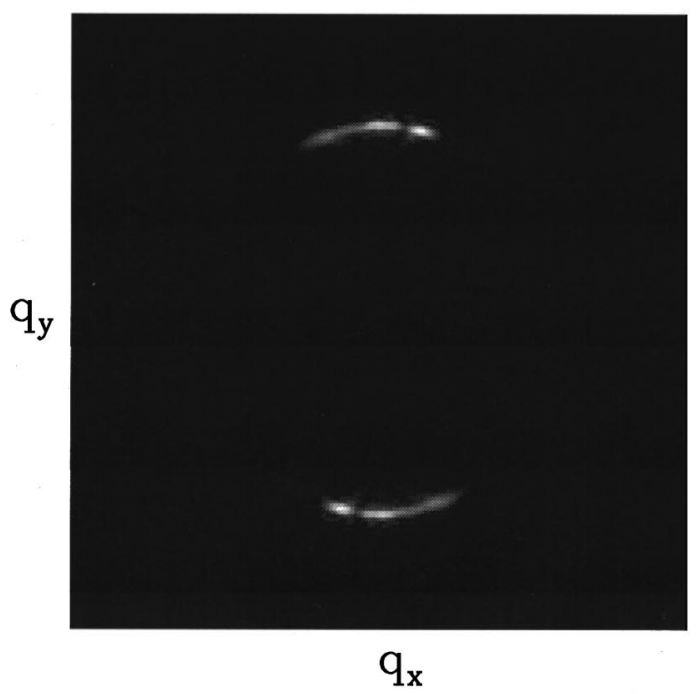

FIG. 4. The (a) near-field and (b) far-field intensity images of the pattern in the absolutely unstable regime are shown $(t=1500)$ in a linear gray-scale. The initial condition is zero for the $\mathrm{FH}$ and the steady-state solution for the pump beam; the random noise of the intensity, $\epsilon_{0}=\epsilon_{1}=1.5 \times 10^{-11}$, is continuously applied. The other parameters as in Fig. $3(*)$, and $\Delta_{0}=1$.

For a normalized pump amplitude $F \simeq 1.06\left(E_{m}=1.5\right)$ (the star in Fig. 3) the system is predicted to be absolutely unstable. In fact, after a transitory in which low intensity, randomly oriented stripes appear, we obtain the NF and FF patterns shown in Figs. 4(a) and 4(b). The NF structure is not static but, because of the advection, drifts upward at the speed $\rho_{1}$. The stripes are mostly oriented parallel to the $x$ axis, in agreement with the prediction that this is the most rapidly spreading mode in the system. In the circular border where the pump intensity drops, stripes bend; this effect might be explained as a boundary effect $[13,18]$. Note that the pattern is occupying the whole region where the pump is present; the stripes are well defined, and almost no imperfections can be observed. These characteristics are very com- a)

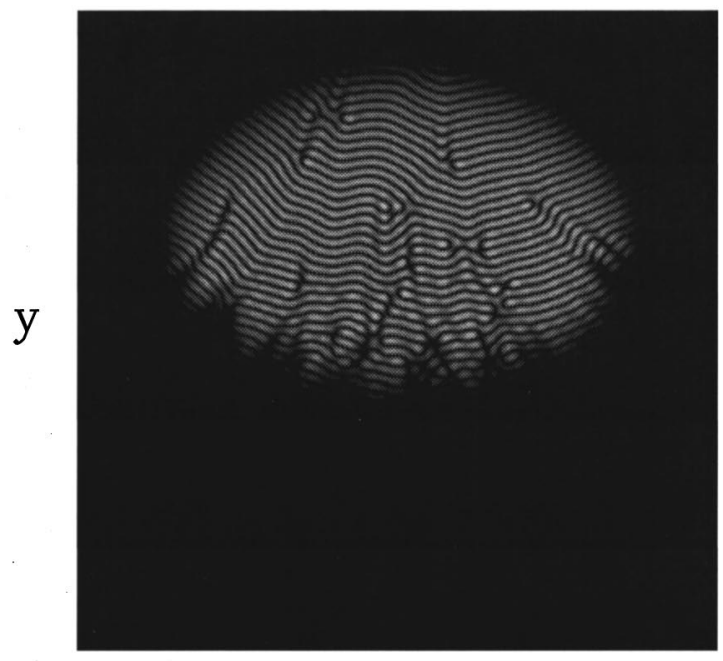

$\mathrm{X}$

b)

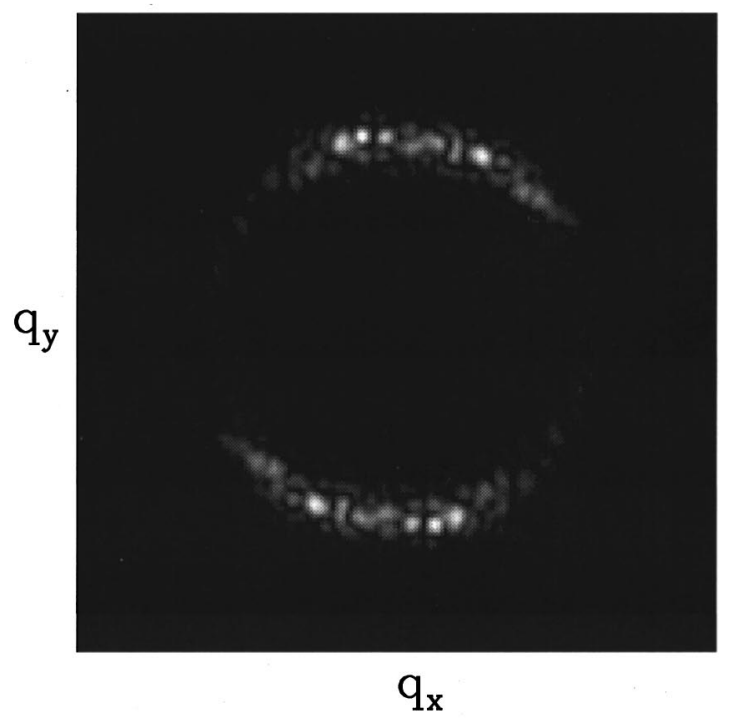

FIG. 5. Same as Fig. 4 for the convectively unstable regime (+ of Fig. 3). The gray scale of (a) is the same as that of Fig. 4(a); the far field in (b) is scaled to its maximum to enhance the visibility.

mon features of the dynamics-sustained patterns.

For a normalized pump amplitude $F \simeq 1.025$ $\left(E_{m}=1.45\right)$ (the cross in Fig. 3) all unstable modes are convective and, after a transitory similar to the previous case, we obtain the structure presented in Figs. 5(a) and 5(b). The pattern is not stationary but drifts upward, as before. However, differently from the absolutely unstable case, observe that the horizontal orientation is less marked, and some imperfections, where stripes bend and turn out not to be horizontal, exist. Moreover, the pattern does not invade all the system, but rather it grows appreciably at a random position well inside the pumping region. As noted [32], noise intensity could be experimentally measured by characterizing the average and dispersion of the spatial delay in the pattern growth, as done in fluid dynamics experiments [31]. All these features are typical of a noise-sustained pattern, and the 


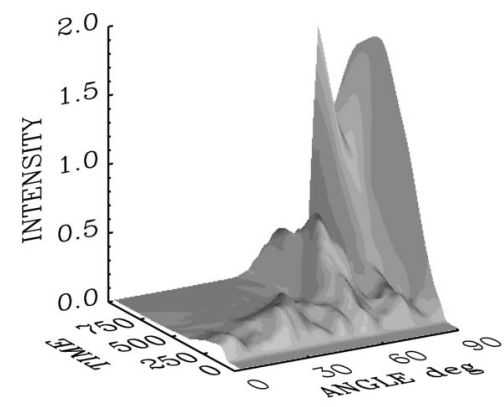

FIG. 6. Intensity distribution for the different spatial modes as a function of the angle $\theta$ and the time as it results from a numerical integration. The intensity content of the modes with $\theta$ closer to zero tends to decrease because of the overwhelming advection, i.e., these modes are convectively unstable. Conversely the most rapidly spreading modes, with larger $\theta$, are absolutely unstable and can grow. The parameters are as for Fig. $3(*)$, except for $\epsilon_{0}=\epsilon_{1}=0$ (no noise); the FH field was initially set to a small intensity, random condition.

analogy with the 1D Kerr case [32] is clear. In this 2D case the pump amplifies a spatial structure generated from the noise at the bottom of the window. While the amplification goes on, the stripes, initially oriented randomly, drift upward and tend to rearrange along the predicted preferred wave vector. If the noise source is turned off, we also observed that the structure disappears in agreement with the prediction.

The observation of the FF also reveals the difference among a noise- and a dynamics-sustained pattern. The FF in Fig. 5(b) is made of two broadened arcs of the ring, and this qualitative behavior does not change even at larger times, since it is due to the fact that noise continues to excite all modes, though some $(\theta \simeq 0)$ leave the system more rapidly than others $(\theta \simeq \pi / 2)$. Conversely, Fig. 4(b) shows much better defined and intense spots close to the modes with $\theta$ $\simeq \pi / 2$. These modes, as we will show below, are the absolutely unstable ones, and their growth overwhelms that of the modes which are convectively unstable.

Thus the results of the numerical integrations indicate a behavior in agreement with the theory with respect to the different behavior of the convectively and absolutely unstable modes. As stated, if the pump amplitude is slightly above the absolute instability threshold, we predicted that both absolutely and convectively unstable modes coexist. The latter group is expected to finally decay in intensity due to the advection while the absolutely unstable modes keep growing for longer times. Eventually, the mode competition should lead to the selection of a single orientation in the pattern [18].

The phenomenon of modal coexistence discussed in Sec. III is well captured in Fig. 6, where we show the total intensity content of the modes $0<\theta<\pi / 2$ in an absolutely unstable situation. Note how a well defined set of modes (for small angles) tends to grow initially but disappears on a larger time scale, due to its unstable but convective nature. Absolutely unstable modes, close to the most rapidly spreading mode $\theta=\pi / 2$, gather a much larger intensity and compete in the fully nonlinear regime. We repeated the numerical integration with different random initial conditions and for different values of the pump amplitude above the pre-

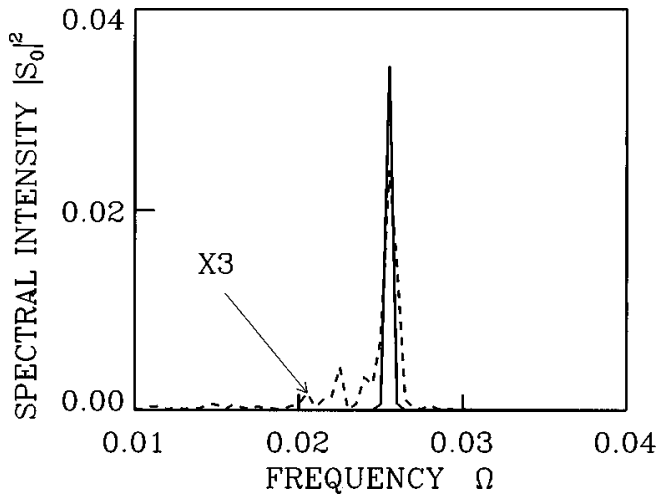

FIG. 7. Spectral intensity of the field amplitude at a fixed spatial position $(0,6.25)$ for $F \simeq 1.0465$ (solid curve) and $F \simeq 1.025$ (dashed curve). The noise level was the same as that of Figs. 4 and 5. The dashed intensity spectrum has been multiplied three times in order to make it visible in the scale of the solid one.

dicted absolute threshold, finding the same qualitative behavior. Unfortunately, a rigorous criterium to identify the critical angle $\theta_{c}$ could not be found from figures like Fig. 6, because of the strong influence of the random initial condition in the dynamics. Nonetheless, we have shown that the stability properties of different spatial modes depend on the relative value of the angle $\theta$. Above a certain critical angle $\theta_{c}$ modes are absolutely unstable while below they are convective. Thus the prediction of Eq. (22) in this regard must be taken qualitatively and not quantitatively, i.e., it is just an analytical indication of the coexistence of convective and absolute modes.

As in Refs. [31,32], the easiest way, also from an experimental viewpoint, to distinguish noise-sustained from deterministic structures is through the time spectral analysis. This is shown in Fig. 7; this picture presents the fast Fourier transform of the waveforms that could be recorded by placing a field detector in the NF, where the pattern reaches its nonlinearly saturated value (in the example we chose the points $x=0$, and $y=6.75)$, both for the convectively $(F \simeq 1.025)$ and absolutely $(F \simeq 1.0465)$ unstable regimes. The difference in the spectral broadening is remarkable; the absolutely unstable pattern spectrum has a variance equal to the frequency resolution of the numerical integration $\left(5 \times 10^{-4}\right)$, while the convective spectrum has about $3 \times 10^{-2}$, i.e. almost two orders of magnitude of difference. The average frequencies are $\Omega=0.0255$ (solid curve) and $\Omega=0.0239$ (dashed curve). The former is in good agreement with the value that can be predicted analytically by means of the results of Sec. III. In fact, through Eqs. (17) and (19) it is also possible to evaluate the (real) wave vector at the absolute instability threshold which is $q_{y} \simeq 1.09$, and thus $\Omega=\rho_{1} q_{a} /(2 \pi)=0.026$. As for the convective case the average frequency is smaller because in this regime modes with $q_{x} \neq 0$ can be still excited (see Fig. 5), and this causes stripe bending and eventually a decrease in the oscillation frequency. Note that this feature is due to the fact that a 2D convective system is considered, and it would be absent otherwise. The transition from one regime to the other is sharp, as we demonstrated in Fig. 8, where the variances of spectra obtained in different spatial positions, as well as their average, are shown. The large spread observed in the variance values is due to the relatively short time of 


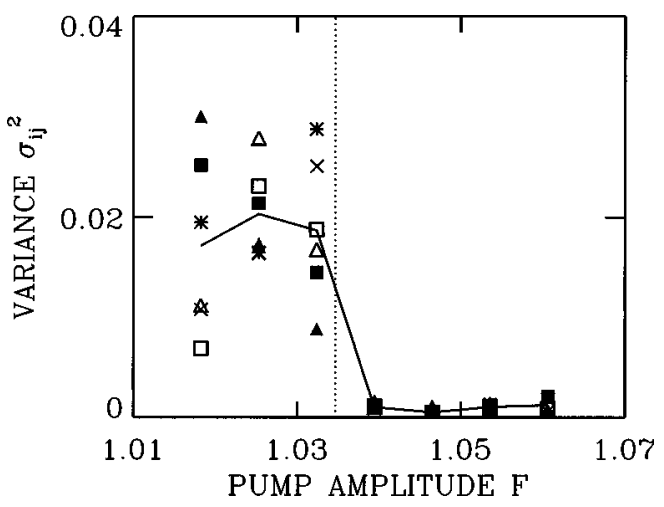

FIG. 8. Variance $\sigma_{i j}^{2}$ of the spectra detected in different positions $\left(x_{i}, y_{j}\right) ; i=1,2$, and 3 and $j=1$, and 2 with $x_{1}=$ $-25, x_{2}=0, x_{3}=25, y_{1}=37.5$, and $y_{2}=68.75$ and their average (solid line). The positions correspond to the symbols: $\left(x_{1}, y_{1}\right)$ filled triangle; $\left(x_{2}, y_{1}\right)$ star; $\left(x_{3}, y_{1}\right)$ triangle; $\left(x_{1}, y_{2}\right)$ filled square; $\left(x_{2}, y_{2}\right)$ cross; $\left(x_{3}, y_{2}\right)$ square. The dotted vertical line indicates the threshold predicted theoretically (see Fig. 3) for the walk-off parameter used $\left(\rho_{1}=0.15\right)$.

integration. The governing equations were in fact in this example integrated for 2000 time units, which is already a highly demanding computational problem for such large 2D systems on a high-speed workstation. The position of the transition from the convectively to the absolutely unstable regime is in very good agreement with the value predicted by the analysis.

Note [Figs. 4(a) and 5(a)] that both the dynamics- and noise-sustained patterns obtained are much more intense than the noise level. In our numerical integration we found up to 11 orders of magnitude of intensity amplification. In this regard it is worth pointing out that, in principle, with an arbitrarily large system (in the direction of the advection) the factor could also be arbitrarily large. Of course, in the case of the OPO the beam size of a real laser pump has physical and technological constraints to be taken into account, as we will discuss below.

\section{FINAL DISCUSSION AND CONCLUSIONS}

We dedicate this final section to a discussion of the problems related to a possible experimental observation of noisesustained structures, and to present our conclusions. The typical parameter values with the current, available materials and technology can be roughly estimated as follows. Nonlinear crystals (see, for example, Refs. [42], Table 19.6-1, and Ref. [52]) can have a nonlinear coefficient $\chi_{\text {eff }}^{(2)}$ up to the order of $100 \mathrm{pm} / \mathrm{V}$. Let us set the refractive index at $n$ $\simeq 3.5$ and a crystal length of $L=8 \mathrm{~mm}$; let us also set the cavity transmittivity at $T=0.05$, which would yield a cavity decay rate of about $0.5 \mathrm{GHz}$ and thus fix the time unit to about 2 ns. The pump laser could be a frequency doubled cw $\mathrm{Nd}$ :YAG (yttrium aluminum garnet), emitting at $532 \mathrm{~nm}$ with an average power of about $15 \mathrm{~W}$ that we suppose to be uniformly distributed over a 4.8-mm-diameter spot, so that the intensity is $I=83 \mathrm{~W} / \mathrm{cm}^{2}$. The amplitude of the input electric field provided is then $E_{0}=\sqrt{2 I / \epsilon_{0} c} \simeq 0.25 \mathrm{kV} / \mathrm{cm}$, ( $\epsilon_{0}$ is the vacuum permittivity) which suffices to bring the OPO to threshold because with these values we can obtain
$F \simeq K_{0}\left|E_{0}\right| \simeq 1$ if $\Delta_{0} \simeq 0$ (small pump detuning and mismatch are conditions which can be easily attained in real experiments). Let us set $a_{1} \simeq 1$ which yields a normalized beam diameter of 80 scaled units, and thus fix the spatial unit $\Delta x$ $=\Delta y$ to about $60 \mu \mathrm{m}$. This diffraction coefficient is larger than that used in the numerical examples of Sec. IV, but noise-sustained structures can be found also in this case. Diffraction affects the wavelength $\lambda_{c}=2 \pi / k_{c}$ of the observed stripes through Eq. (12) as well as the threshold of the absolute instability. In particular, the larger $a_{1}$, the smaller the threshold. However, with a walk-off angle on the order of $0.1 \mathrm{mrad}$ we also obtain $\rho_{1} \simeq 0.27$, i.e., larger than that used in the numerical example shown. If the FH detuning is still set to $\Delta_{1}=-0.25$, the region of the convective instability is the same as for $a_{1}=0.25, \rho_{1}=0.15$. In fact, Eq. (21) [and Eq. (12) for $\Delta_{1}$ fixed] shows that the position of the absolutely unstable threshold actually depends on the ratio $\rho_{1}^{2} / a_{1}$, which is the same for the numerical resolution and this estimation. In particular, this example yields that the absolute and convective threshold are separated by $0.525 \mathrm{~W}$. Finally, in this example, seven stripes can be accommodated in the pumping region. Therefore, an experimental observation of noisesustained structures in OPO's seems feasible, on the basis of the theory, the numerical resolutions, and the available data on realizable experimental set-up.

As a final remark, we want to stress the main reasons that justify this statement. The walk-off is an intrinsic effect of parametric down-conversion as soon as birefringent media are used [53]. The convective regime is the first to appear above threshold; there is no particular power requirement except that of being at the signal (and thus pattern) generation threshold. The structure is as intense as the one observed in the absolutely unstable regime, so that it can be directly detected, noise having been amplified many orders of magnitude. The direct detection of the NF and FF shows clear fingerprints of the nature of the pattern, and thus allows one to distinguish when a noise- or dynamics-sustained pattern is observed. Finally, even if direct observation is not possible, the time spectral analysis is straightforwardly simple, since it relies on the detection of a well developed optical field intensity oscillation [54]. It is also worth mentioning that in the case of drift instabilities with Kerr-like nonlinearities, the existence of "broad frequency distributions" in the spectral analysis [6], "small oscillations confused with noise" [9], and "random pattern precursors" [55] have been already reported but not fully investigated and explained.

In conclusion we have theoretically demonstrated the existence of noise-sustained structures in a model of type I, degenerate optical parametric oscillators for negative signal detuning. We analyzed in detail the formation of transversal two-dimensional structures in the optical field intensity. Special features appear, due to the two-dimensional modeling and the presence of the walk-off. The latter effect causes the instability to be convective, just above threshold, and thus noise-sustained structures can be found in this regime. We identified the main features that help to distinguish the dynamics-sustained structure in the absolutely unstable regime from the noise-sustained pattern in the convectively unstable regime. A simple direct detection of the near and far fields or a time-spectral analysis are apt to discern the nature of the pattern under investigation. 
The walk-off also breaks the rotational symmetry of the system, causing a preferential orientation of the pattern because of the advection-spreading balance mechanism, which is peculiar of a convective system. This symmetry breaking also manifests itself in the coexistence of absolutely and convectively unstable modes for pump amplitudes slightly above the absolute instability threshold.

It is well known that OPO's are devices suitable for the generation of nonclassical states of light. Here we demonstrated that quantum noise can be amplified several orders of magnitude, and can in principle give rise to a macroscopic pattern in the convective regime. Thus convective noise- sustained structures may become a paradigmatic example to search for quantum statistics and quantum correlations in macroscopic, spatially structured optical systems.

We point out that the results of this analysis, as well as the methods used, are very general, and can be used as a reference to study pattern formation in convective, 2D systems, in other branches of physics.

\section{ACKNOWLEDGMENTS}

This work was supported by QSTRUCT (Project No. ERB FMRX-CT96-0077). Financial support from DGICYT (Spain) under Project No. PB94-1167 is also acknowledged.
[1] Transverse Effects in Nonlinear Optical Systems, edited by N. Abraham and W. J. Firth, special issue of J. Opt. Soc. Am. B 7, 948 (1990); Nonlinear Optical Structures, Patterns, Chaos, edited by L. A. Lugiato and M. S. El Naschie, special issue of Chaos Solitons Fractals 4, 1251 (1994), and references therein.

[2] L. A. Lugiato and R. Lefever, Phys. Rev. Lett. 58, 2209 (1987).

[3] W. J. Firth, A. J. Scroggie, G. S. McDonald, and L. A. Lugiato, Phys. Rev. A 46, R3609 (1992).

[4] G. D’Alessandro and W. J. Firth, Phys. Rev. Lett. 66, 2597 (1991); Phys. Rev. A 46, 537 (1992).

[5] M. Tamburrini, M. Bonavita, and S. Wabnitz, Opt. Lett. 18, 855 (1993)

[6] G. Grynberg, Opt. Commun. 109, 483 (1994); A. Petrossian, L. Dambly, and G. Grynberg, Europhys. Lett. 29, 209 (1995).

[7] S. Residori, P. L. Ramazza, E. Pampaloni, S. Boccaletti, and F. T. Arecchi, Phys. Rev. Lett. 76, 1063 (1996).

[8] L. A. Lugiato and C. Oldano, Phys. Rev. A 37, 3896 (1988).

[9] P. La Penna and G. Giusfredi, Phys. Rev. A 48, 2299 (1993).

[10] T. Ackerman, Yu. A. Logvin, A. Heuer, and W. Lange, Phys. Rev. Lett. 75, 3450 (1995).

[11] C. Etrich, U. Peschel, and F. Lederer, Phys. Rev. E 56, 4803 (1997).

[12] K. Staliunas, Opt. Commun. 91, 82 (1992); J. Mod. Opt. 42, 1261 (1995).

[13] G.-L. Oppo, M. Brambilla, and L. A. Lugiato, Phys. Rev. A 49, 2028 (1994); G.-L. Oppo, M. Brambilla, D. Camesasca, A. Gatti, and L. A. Lugiato, J. Mod. Opt. 41, 1151 (1994).

[14] J. B. Geddes, J. V. Moloney, E. M. Wright, and W. J. Firth, Opt. Commun. 111, 623 (1994); A. G. Scroggie and W. J. Firth, Phys. Rev. A 53, 2752 (1996).

[15] G. Grynberg, A. Maitre, and A. Petrossian, Phys. Rev. Lett. 72, 2379 (1994).

[16] A. Aumann, E. Büthe, Yu. A. Logvin, T. Ackemann, and W. Lange, Phys. Rev. A 56, R1709 (1997).

[17] M. Hoyuelos, P. Colet, M. San Miguel, and D. Walgraef, Phys. Rev. E (to be published); M. Hoyuelos, P. Colet, and M. San Miguel, Phys. Rev. E (to be published).

[18] M. C. Cross and P. C. Hohenberg, Rev. Mod. Phys. 65, 851 (1993).

[19] S. Longhi, J. Mod. Opt. 43, 1089 (1996); 43, 1569 (1996); Phys. Rev. A 53, 4488 (1996); S. Longhi and A. Geraci, ibid. 54, 4581 (1996).

[20] G. J. de Valcarcel, K. Staliunas, E. Roldan, and V. J. Sanchez-
Morcillo, Phys. Rev. A 54, 1609 (1996); V. J. SanchezMorcillo, E. Roldan, G. J. de Varcarcel, and K. Staliunas, ibid. 56, 3237 (1997).

[21] R. A. Fuerst, D.-M. Baboiu, B. Lawrence, W. E. Torruellas, G. I. Stegeman, S. Trillo, and S. Wabnitz, Phys. Rev. Lett. 78, 2756 (1997).

[22] L. A. Wu, H. J. Kimble, J. Hall, and H. Wu, Phys. Rev. Lett. 57, 2520 (1986); L. A. Wu, M. Xiao, and H. J. Kimble, J. Opt. Soc. Am. B 4, 1465 (1987).

[23] M. D. Reid and P. D. Drummond, Phys. Rev. Lett. 60, 2731 (1988); J. Mertz, T. Debuisschert, A. Heidmann, C. Fabre, and E. Giacobino, Opt. Lett. 16, 1234 (1991); P. G. Kwiat, K. Mattle, H. Weinfurter, A. Zeilinger, A. V. Sergienko, and Y. Shih, Phys. Rev. Lett. 75, 4337 (1995).

[24] L. A. Lugiato, S. Barnett, A. Gatti, I. Marzoli, G. L. Oppo, and H. Wiedemann, Coherence and Quantum Optics VII (Plenum, New York, 1996), p. 5.

[25] G. Grynberg and L. A. Lugiato, Opt. Commun. 101, 69 (1993); L. A. Lugiato and G. Grynberg, Europhys. Lett. 29, 675 (1995).

[26] A. Gatti and L. A. Lugiato, Phys. Rev. A 52, 1675 (1995).

[27] L. A. Lugiato and I. Marzoli, Phys. Rev. A 52, 4886 (1995).

[28] A. Gatti, H. Wiedemann, L. A. Lugiato, I. Marzoli, G.-L. Oppo, and S. M. Barnett, Phys. Rev. A 56, 877 (1997); A. Gatti, L. A. Lugiato, G.-L. Oppo, R. Martin, P. Di Trapani, and A. Berzanskis, Opt. Express. 1, 21 (1997).

[29] I. Marzoli, A. Gatti, and L. A. Lugiato, Phys. Rev. Lett. 78, 2092 (1997).

[30] I. Rehberg, S. Rasenat, M. de la Torre Juárez, W. Schöpf, F. Hörner, G. Ahlers, and H. R. Brand, Phys. Rev. Lett. 67, 596 (1991); M. Wu, G. Ahlers, and D. S. Cannell, ibid. 75, 1743 (1995).

[31] K. L. Babcock, G. Ahlers, and D. S. Cannell, Phys. Rev. Lett. 67, 3388 (1991); Phys. Rev. E 50, 3670 (1994); A. Tsameret and V. Steinberg, Phys. Rev. Lett. 67, 3392 (1991); Phys. Rev. E 49, 1291 (1994).

[32] M. Santagiustina, P. Colet, M. San Miguel, and D. Walgraef, Phys. Rev. Lett. 79, 3633 (1997).

[33] R. J. Deissler, Stat. Phys. 40, 376 (1985); 54, 1459 (1989); Physica D 56, 303 (1992).

[34] M. Haelterman and G. Vitrant, J. Opt. Soc. Am. B 9, 1563 (1992); G. Vitrant and J. Danckaert, Chaos Solitons Fractals 4, 1369 (1994).

[35] E. Infeld and G. Rowlands, Nonlinear Waves, Solitons and 
Chaos (Cambridge University Press, London, 1990), Chap. 3, and references therein.

[36] G. G. Luther and C. J. McKinstrie, J. Opt. Soc. Am. B 7, 1125 (1990)

[37] N. Bloembergen, Nonlinear Optics (Benjamin, Reading, MA, 1965).

[38] S. A. Akhmanov, A. P. Sukhorukov, R. V. Khokhlov, Zh. Eksp. Teor. Fiz. 50, 474 (1996) [Sov. Phys. JETP, 23, 316 (1966)].

[39] Y. R. Shen, The Principles of Nonlinear Optics (Wiley, New York, 1984).

[40] J. P. Seipenbusch, T. Ackemann, B. Schäpers, B. Berge, and W. Lange, Phys. Rev. A 56, R4401 (1997).

[41] The momentum conservation is written for a so called collinear (or quasicollinear) phase-matching where wave vectors are parallel (or quasiparallel), and thus $\kappa_{p, s, i}$ represent the moduli.

[42] B. E. A. Saleh and M. C. Teich, The Fundamentals of Photonics (Wiley, New York, 1991).

[43] G. J. Hall, M. Ebrahimzadeh, A. Robertson, G. P. Malcom, and A. I. Ferguson, J. Opt. Soc. Am. B 10, 2168 (1993).

[44] See, for example, A. V. Smith, W. J. Alford, T. D. Raymond, and M. S. Bowers, J. Opt. Soc. Am. B 12, 2253 (1995); Y. Tang, C. F. Rae, C. Rahlff, and M. H. Dunn ibid. 14, 3442 (1997).

[45] W. E. Torruellas, Z. Wang, D. J. Hagan, E. W. Van Stryland, G. I. Stegeman, L. Torner, and C. R. Menyuk, Phys. Rev. Lett. 74, 5036 (1995); W. E. Torruellas, G. Assanto, B. L. Lawrence, R. A. Fuerst, and G. I. Stegeman, Appl. Phys. Lett. 68, 1449 (1996).

[46] P. D. Drummond, K. J. McNeil, and D. F. Walls, Opt. Acta 27, 321 (1980).
[47] L. S. Hall and W. Heckrotte, Phys. Rev. 166, 120 (1968); T. H. Stix, Waves in Plasmas (AIP, New York, 1992), Chap. 9.

[48] Neglecting the walk-off, for $\Delta_{1}>0$ the trivial steady state is unstable for homogeneous perturbations, and the field evolves to a uniform solution so no pattern is formed. However, the walk-off can affect the selection mechanism and an inhomogeneous pattern can also be formed for small positive signal detunings. This process is not related to the convective instability phenomenon and the noise-sustained structures, and is described in Ref. [49].

[49] R. Tagg, W. S. Edwards, and H. L. Swinney, Phys. Rev. A 42, 831 (1990).

[50] G. Dee and J. Langer, Phys. Rev. Lett 50, 383 (1983); W. Van Saarloos, Phys. Rev. A 37, 211 (1988).

[51] M. Santagiustina, P. Colet, M. San Miguel, and D. Walgraef, Opt. Lett. (to be published).

[52] J.-Y Zhang, J. Y. Huang, Y. R. Shen, Laser Science and Technology (Harwood, Amsterdam, 1995), Vol. 19; C. L. Tang and L. K. Cheng, Laser Science and Technology (Harwood, Amsterdam, 1995), Vol. 20.

[53] Recently, birefringent materials with large nonlinearities and no walk-off $\left(\mathrm{LiNbO}_{3}, \mathrm{KTA}\right)$ have been made available. The walk-off effect disappears when the propagation in the crystal takes place along the principal axes. However, even in this case a weak walk-off, due to experimental crystal misalignment, should be present, and this is enough to obtain a convective regime, as shown.

[54] For the example given, the oscillation frequency would be about $25 \mathrm{MHz}$.

[55] F. Castaldo, D. Paparo, and E. Santamato, Opt. Commun. 143, 57 (1997). 